\title{
The development of a new hollow cylinder apparatus for investigating the effects of principal stress rotation in soils
}

\author{
D. W. HIGHT, A. GENS and M. J. SYMES (1983). Géotechnique 33, No. 4, 355-383
}

and

\section{Undrained anisotropy and principal stress rotation in saturated sand}

\author{
M. J. P. R. SYMES, A. GENS and D. W. HIGHT (1984). Géotechnique 34, No. 1, 11-27
}

\begin{abstract}
Dr A. Saada and Dr P. Puccini, Case Western Reserve University

The Authors have made thorough observations of the behavior of anisotropic sands under undrained conditions and have attempted to extend the critical state theory to explain the soil's behavior using results obtained from hollow cylinder tests. The Writers would like to make a few remarks regarding the use of the hollow cylinder as well as to contribute some data on the anisotropy of sands.
\end{abstract}

On the stress distribution in the hollow cylinder and the use of the coefficient $\mathrm{b}$

The coefficient $b$, under the name of $\lambda$, seems to have been used first by Habib (1953) in his doctoral thesis. Inspired by Lode's coefficient

$$
\mu=\frac{\sigma_{2}-\frac{1}{2}\left(\sigma_{1}+\sigma_{3}\right)}{\frac{1}{2}\left(\sigma_{1}-\sigma_{3}\right)}
$$

it gives the relative magnitude of $\sigma_{2}$ with respect to $\sigma_{1}$ and $\sigma_{3}$. Therefore, by itself, it does not describe the influence of $\sigma_{2}$ independently from $\sigma_{1}$ and $\sigma_{3}$. This coefficient was introduced and used in conjunction with isotropic materials. In the extensive literature on anisotropic materials, the Writers are not aware that it has ever been used. Each of $\sigma_{1}, \sigma_{2}$ and $\sigma_{3}$ affect anisotropic materials differently depending on their inclination on the axes of material symmetry. Thus, not only is it a parameter that by itself cannot describe the effect of $\sigma_{2}$, but its use is also questionable in the study of anisolropic materials.

The hollow cylinder apparatus has been used to investigate the properties of clay and sand materials for nearly 20 years at Case Western Reserve University. Such devices always offer the possibility of using different inside and outside pressures, in addition to applying axial and torsional stresses. While attempting to take advantage of all the experimental possibilities that the hollow cylinder configtaration offers, the senior Writer conducted analyses similar to the analyses conducted by the Authors, in addition to a three-dimensional photoelastic analysis by the frozen stress technique. The relevant questions are as follows. Should one knowingly create a non-uniformity in the normal radial and normal circumferential stresses and then proceed to assume the applicability of a mathematical model to show that working with averages is reasonable? Also, is it logical to justify the acceptability of a test configuration using an assumed 'isotropic' elasto-plastic model and then to use this configuration to establish the validity of models supposed to describe 'anisotropic' materials? The Writers do not believe so. It should be remembered that one of the reasons for which the hollow cylinder device was criticized for so long is the fact that the nonuniformities across the thickness cast a doubt on the value of the collected data. Here the problem is also complicated by the fact that the material is anisotropic. Different $\sigma_{r}$ and $\sigma_{\theta}$ within the thickness of the sample result in different tendencies for deformation in the axial direction, and thus to non-uniform axial stresses. If one starts with a transversely isotropic material and then applies different inside and outside pressures, this kind of symmetry changes to one of the orthotropic type, whose degree varies radially. These transformations become more pronounced as the torsional shears are applied to the cylinder. The commonly made assumption that the material remains transversely isotropic becomes impossible to support with different $\sigma_{r}$ and $\sigma_{\theta}$. 
When the same pressure is applied inside and outside the cylinder in a cell, this cell pressure is $\sigma_{2}$. The major and minor principal stresses are controlled by the combination of axial and torsional stresses. As noticed by the Authors, $b=$ $\sin ^{2} \alpha$ and each inclination of the major principal stress corresponds to a given $b$. The Writers have conducted parallel series of tests with corresponding inclinations of principal stress; in one series the cell pressure, i.e. $\sigma_{2}$, was kept constant and in the other the mean stress was kept constant. Thus for the same $b, \sigma_{2}$ was constant in one series and continuously variable in the other series. These tests, which are described at length in the references by Saada and coworkers that are cited by the Authors in their paper, are but one example that $b$ does not uniquely describe $\sigma_{2}$, and vice versa. It appears to the Writers that the main reason that the Authors use different inner and outer pressures is to change $b$. Is it worth sacrificing uniformity and casting doubts on the data just to use such an unrepresentative parameter? Why not find another parameter that does not require a different inner and outer pressure? Why not study the effects of $\sigma_{2}$ by looking at $\sigma_{2}$ directly rather than through $b$ ? Since the Authors are using $\alpha$ as a parameter why use $b$ which can be related to $\alpha$ ?

The Writers submit that the price that is being paid for the use of $b$ is too high. With different inner and outer pressures the hollow cylinder testing device is not much of an improvement over the direct simple shear device since it involves nearly as many non-uniformities in the stress distribution, with the added dangerous impression that more refined boundary measurements give accurate directional properties.

On the initial or inherent anisotropy of sands

For the last three years an intensive program of research on the mechanical properties of

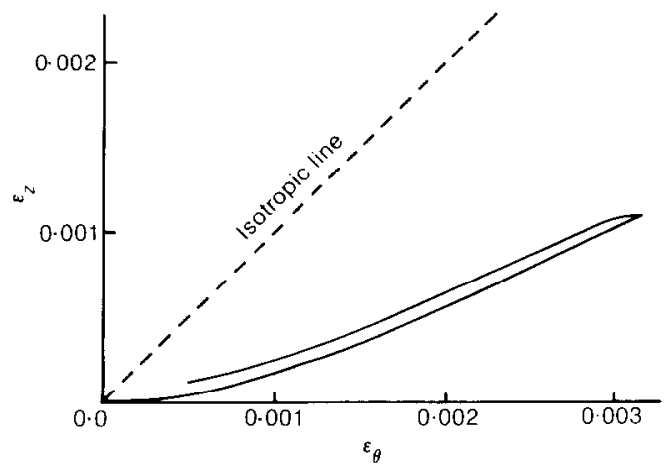

Fig. 1. Axial and circumferential strains in spherical consolidation naturally deposited sand has been in progress at Case Western Reserve University. The results of some of the investigations were presented at the CNRS international colloquium held in Grenoble in June 1983 and will appear in the proceedings of the colloquium. The graphs in this discussion are taken from the paper in the proceedings.

Tests were conducted on a uniform sand $\left(C_{\mathrm{u}}=1.64\right)$ known as Reid Bedford sand at a medium relative density and a void ratio of $0 \cdot 67$. Thin long hollow cylinders with $7 \cdot 1 \mathrm{~cm}$ outside diameter, $5 \cdot 1 \mathrm{~cm}$ insider diameter and a length of approximately $12.7 \mathrm{~cm}$ were prepared by pluviation and subjected 'drained' to a variety of stress paths. In all the static tests, axial torsional and cell stresses were varied maintaining a constant ratio so that $\alpha$ was always constant for a given test. Fig. 1 shows the strains when the hollow cylinder was subjected to spherical stresses varying from $40 \mathrm{kPa}$ to $600 \mathrm{kPa}$ and back to $40 \mathrm{kPa}$. The rebound is nearly elastic, though non-linear and inherent anisotropy prominently displayed by a ratio of $\varepsilon_{\theta} / \varepsilon_{z}$ between $1 / 6$ and $1 / 3$ approximately. A ratio of unity corresponds to an isotropic response. Fig. 2 shows the axial and circumfer-

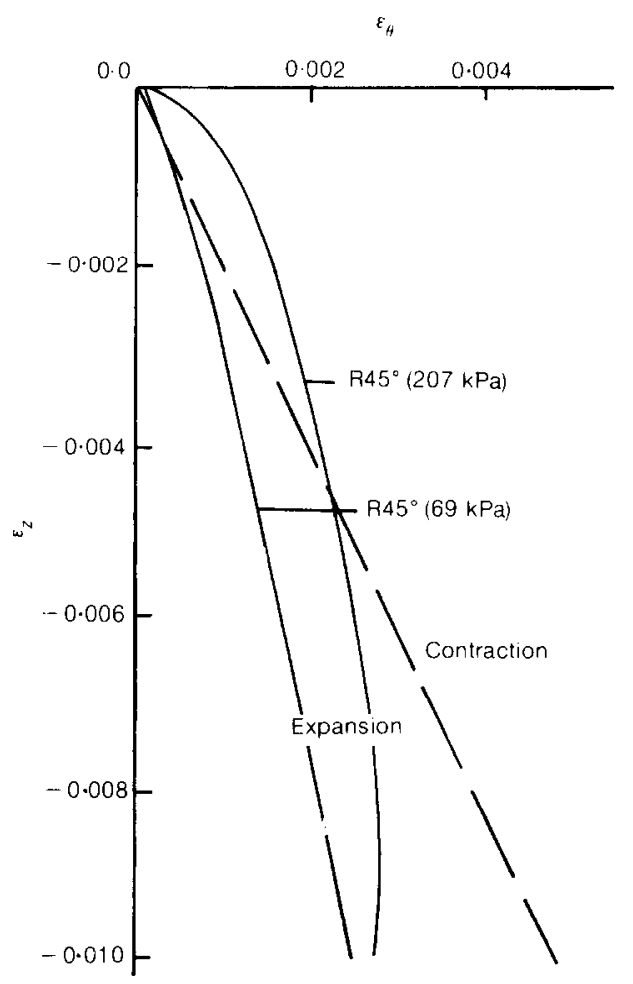

Fig. 2. Axial and circumferential strains in pure torsion 
ential strains for two samples, one under a spherical effective stress of $69 \mathrm{kPa}$ and the other under $207 \mathrm{kPa}$, both being subjected to pure torsion to failure. Under $69 \mathrm{kPa}$ the volume of the sample constantly increases with axial elongation and lateral contraction, the latter being smaller than the vertical contraction. Under $207 \mathrm{kPa}$ the volume of the sample first decreases, with the lateral contraction being larger than the axial elongation, then increases with the reverse being true. Each of the two curves in Fig. 2 shows the prominence of the dilatancy and Poynting-Weissenberg effects, both characteristic of non-linear behavior. The changes in the slopes along the two curves are indicative of the changes in anisotropy induced by the applied torsional stresses. Looking at both Figs 1 and 2 one notices the very large volume changes under simple shear compared with those taking place under spherical stress and the substantial differences in the strains taking place in the axial direction when compared in magnitude with those taking place in the circumferential direction. It is felt that by looking at drained tests one can better judge inherent or initial anisotropy and have an idea about how it varies as the state of stress changes.

Trying to separate initial from induced anisotropy is an interesting but not very useful exercise, since one can create a new particle arrangement with each state of strain and an 'induced anisotropy is seen as an inherent anisotropy by every new state of stress to which the sample is subjected'. However, it is interesting to know how much the initial anisotropy is changed by the applied stress systems and how those changes affect the mechanical behavior. Fig. 3 shows stress-strain curves for two specimens. One specimen was tested under static conditions and an inclination of the major principal stresses of $31.75^{\circ}$. The other sample was tested under the same conditions but after it had been disturbed by 25 cycles of very severe dynamic loading with complete reversal of both the axial and the torsional stresses. The dynamic loading was sinusoidal and at the point of stress reversal the major principal stress jumped to $58.25^{\circ}$ while the vertical and the minor principal stress jumped to $31.75^{\circ}$. The amplitude of the stresses were such that the axial stress was approximately $75 \%$ of the failure value in extension and the torsional stress was proportional to it, so that the inclinations were maintained constant during each half of the cycle. In Fig. 3(a) one notices that the volume change curve of the disturbed sample falls below the volume change curve of the undisturbed sample, as it should, since cyclic loading even under large strain re-
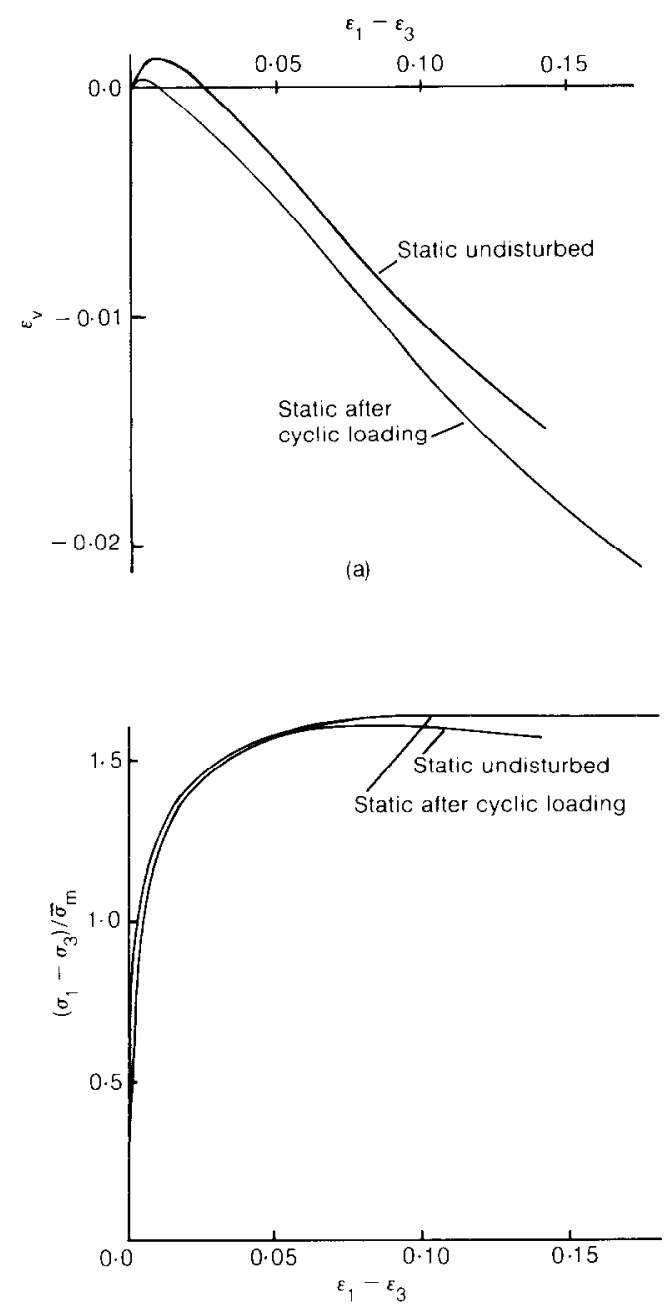

(b)

Fig. 3. Stress-strain relations for $\alpha=31.75^{\circ}$

sults in contraction (beyond one or two complete cycles). In Fig. 3(b), however, the two strength curves are quite close. There is more initial rigidity in the disturbed sample but overall the strength behavior is comparable in spite of the small difference in void ratio between the two samples. It is surmised that it is difficult to eradicate the initial anisotropy of the material even with such a severe treatment. Similar results were obtained from specimens under a wide variety of consolidation pressures and stress paths.

Regarding the existence of a state boundary surface the Writers wish that the Authors had conducted a few tests in which the specimens are made to elongate rather than to contract, i.e. to deal with inclinations $\alpha$ between $45^{\circ}$ and $90^{\circ}$. It 


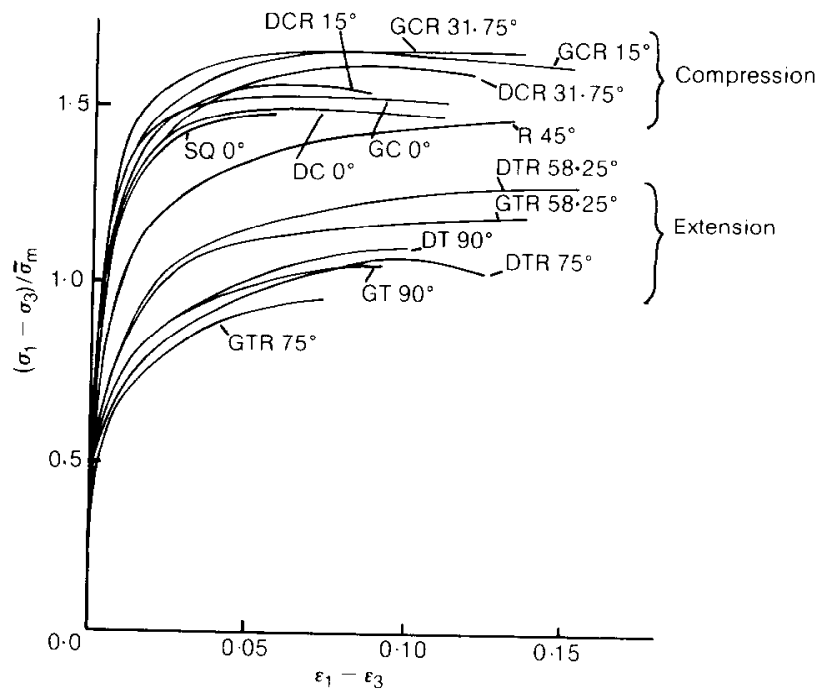

Fig. 4. Normalized stress-strain relations for $\bar{\sigma}_{\mathrm{c}}=270 \mathrm{kPa}$

is within this range that critical state soil mechanics meets its hardest challenge. On the basis of extensive test results on sands and clays the Writers feel that the critical statc conccpt provides an excellent framework and a new terminology within which soil mechanics can grow and develop, but they agree with Bishop's conclusion that the use of $M$ for describing soil behavior is definitive only if it is coupled with a reference to the type of test from which the test data were obtained. Trying to extend this concept to anisotropic materials without major modifications will be a challenge. Fig. 4 shows stress-strain curves for hollow cylinders tested with various inclinations of the major principal stress on the axis of symmetry. On this figure, the label DCR $15^{\circ}$ for example refers to a compression and a torsion such that $\alpha=15^{\circ}$ and the cell pressure remains constant $\left(\sigma_{2}\right.$ is constant); GTR $75^{\circ}$ refers to an extension and a torsion such that $\alpha=75^{\circ}$ and the mean stress remains constant $\left(\sigma_{2}\right.$ varies); $\mathrm{R}$ refers to pure torsion. Fiven though they are all drained tests they show the great difference that exists between the group in which axial compression takes place and the group in which axial extension takes place. The material is quite rigid (or brittle) in compression, but quite ductile in extension. Under such circumstances it is difficult to see a state boundary surface (at least at this stage).

\section{Dr A. K. Jamal, Comell University}

For the boundary condition $p_{\mathrm{o}}=p_{\mathrm{i}}$ in the hollow cylinder test, the radial stress $\sigma_{\mathrm{r}}$ is uniformly distributed across the wall thickness of the test specimen and therefore the radial stress $\sigma_{r}$ is equal to the circumferential stress $\sigma_{\theta}$ irrespective of the shear stress $\tau_{\theta z}$. Then the circumferential strain $\varepsilon_{\theta}$ can also be assumed to be uniform and from the compatibility of strains

$$
\varepsilon_{r}-\varepsilon_{\theta}=r \frac{\mathrm{d} \varepsilon_{\theta}}{\mathrm{d} r}
$$

it follows that the radial strain $\varepsilon_{r}$ and the circumferential strain $\varepsilon_{\theta}$ are equal.

The Writer's discussion is concerned with an inquiry into the strains $\varepsilon_{r}$ and $\varepsilon_{\theta}$ in the hollow cylinder test.

If the test cylinder is considered as an element and isotropy is assumed, the octahedral shear strain $\gamma_{\text {oct }}$ may, for convenience here, be referred to the component of the major principal strain $\varepsilon_{1}$ involved in causing purely shear deformation expressed as $\left|\varepsilon_{1}-\varepsilon_{\mathrm{v}} / 3\right|$. $^{*}$ Fig. 5(a) has thus been constructed to show the relationship of $\gamma_{\text {oct }}$ to $\left|\varepsilon_{1}-\varepsilon_{\mathrm{v}} / 3\right|$ for each of the tests E1, E2 and E3. The strain values of $\varepsilon_{1}, \varepsilon_{\mathrm{v}}$ and $\gamma_{\text {oct }}$ were read from the Authors' Figs 32 and 34 corresponding to test stage $\mathrm{B}\left(\sigma_{1} / \sigma_{3}=3 \cdot 5\right)$ and for the total stress path along $\mathrm{BF}$ to the stress ratio level $\sigma_{1} / \sigma_{3} \approx 4.4$ (end value for $\mathrm{E} 3$ ). For the $\mathrm{E} 2$

\footnotetext{
* Similarly for the intermediate and the minor principal strains the component of the strain causing shear deformation only can be written as $\left|\varepsilon_{z}-\varepsilon_{\mathrm{v}} / 3\right|$ and $\left|\varepsilon_{3}-\varepsilon_{\mathrm{v}} / 3\right|$ respectively, whence

$$
\varepsilon_{1}+\varepsilon_{2}+\varepsilon_{3}=\varepsilon_{\mathrm{v}}
$$
}

and

$$
\gamma_{\mathrm{oct}}=2\left[\left(\varepsilon_{1}-\varepsilon_{2}\right)^{2}+\left(\varepsilon_{1}-\varepsilon_{3}\right)^{2}+\left(\varepsilon_{2}-\varepsilon_{3}\right)^{2}\right]^{1 / 2} / 3
$$


test, the points plotted correspond to the stress ratio $\sigma_{1} / \sigma_{3}$ values of 2,3 and $3 \cdot 5$, i.e. to the triaxial compression stage of this test up to the point $C$ *

The test values of $\gamma_{\text {oct }}$ in Fig. 5(a) indicate that the intermediate and the minor principal strains $\varepsilon_{2}$ and $\varepsilon_{3}$ are unequal in the respective hollow cylinder tests. The intermediate principal strain $\varepsilon_{2}$ which is equal to the radial strain $\varepsilon_{r}$ can be determined by solving the strain equilibrium equations of $\varepsilon_{\mathrm{v}}$ and $\gamma_{\mathrm{oct}}$. Then by referring the volumetric strain $\varepsilon_{\mathrm{v}}$ to the $r, \theta, z$ co-ordinate axis the circumferential strain $\varepsilon_{\theta}$ can be deduced from the known values of the strains $\varepsilon_{z}$ and $\varepsilon_{r}$. The computed values of $\varepsilon_{r}$ and $\varepsilon_{\theta}$ are also plotted in Fig. 5(a) and it is seen that $\varepsilon_{\mathrm{r}}>\varepsilon_{\theta}$. Because of the small strain values in the initial stages of the E2 test the $\varepsilon_{r}$ and $\varepsilon_{\theta}$ values were not computed but at point $C$ the circumferential strain $\varepsilon_{\theta}$ is about one-half of the radial strain $\varepsilon_{r}$. For the test path $\mathrm{CB}$ during the application of $\tau_{\theta z}$ at constant maintained $\sigma_{1} / \sigma_{3}$ the test specimen underwent volumetric compression and the computed incremental strains $\varepsilon_{\theta}$ and $\varepsilon_{r}$ are of the order of $0 \%$ and $0.29 \%$ for $\mathrm{d} \varepsilon_{\theta}$ and $\mathrm{d} \varepsilon_{r}$ respectively.

Since the axial strain $\varepsilon_{\mathrm{z}}$ is an extension strain of unequal magnitudes in the F1 and E3 tests as well as in the E2 test for the stress path BF, a comparison of the strain pattern in these tests was made in terms of the strain ratio $\varepsilon_{r} / \varepsilon_{1}$ and $\varepsilon_{\theta} / \varepsilon_{1}$ as shown in Fig. 5(b). A comparison with the Writer's investigations cited in the Authors' paper on hollow cylinder specimens for which $\sigma_{1}=\sigma_{2}>\sigma_{3}$ and $\sigma_{1}=\sigma_{2}=\sigma_{3}$ shows clearly the trend for $\varepsilon_{r} \neq \varepsilon_{\theta}$ and conforms to the theoretical deduction of an inequality between the strains $\varepsilon_{r}$ and $\varepsilon_{\theta}$ with a volume change (Jamal, 1966).

The new hollow cylinder apparatus marks a major advance in the experimental technique of investigating soil specimens under versatile test conditions. The exact measurement of the relative radial, axial and shear displacements made by internal scnsors is essential to the study of mechanics of dilatant media.

\section{REFERENCE}

Jamal, A. K. (1966). The distribution of radial stress in the triaxial strength test of a cohesionless, granular soil. PhD thesis, Cornell University.

\footnotetext{
* The hollow cylinder specimen in this test under triaxial compression exhibits 'brittle' behavior as seen in the $\varepsilon_{\mathrm{z}}$ and $\varepsilon_{\mathrm{v}}$ values attained, with lower compressive strength compared with that of the solid cylinder specimen.
}

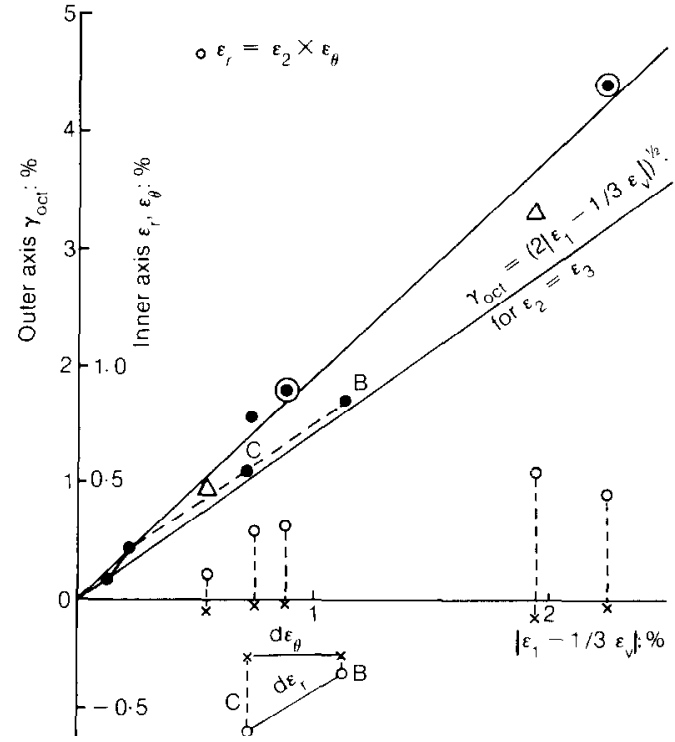

(a)

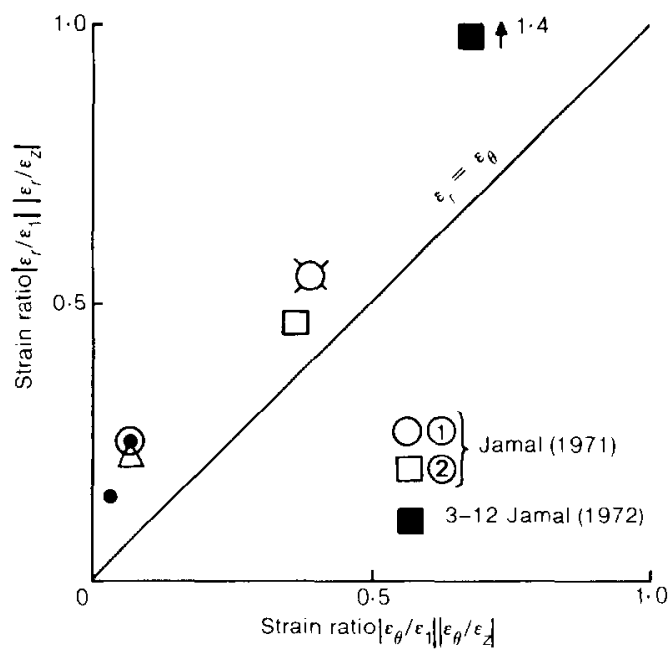

(b)

Fig. 5. (a) Variation in the strains $\gamma_{\alpha \uparrow,}, \varepsilon_{\mathrm{r}}$ and $\varepsilon_{\theta}$ and (b) strain ratios in the hollow cylinder specimens

\section{Authors' reply}

Dr Saada and Dr Puccini raise a number of important issues which relate to the two recent papers published by the Authors.

Firstly, they question the use of $b$ ( $==$ $\left.\left(\sigma_{2}-\sigma_{3}\right) /\left(\sigma_{1}-\sigma_{3}\right)\right)$ as a controlling parameter for conducting hollow cylinder tests. Since the interpretation of such tests is made in terms of a stress tensor with four non-zero components 
$\left(\sigma_{r}, \sigma_{\theta}, \sigma_{z}\right.$ and $\left.\tau_{\theta z}\right)$, the use of any set of four parameters which are independent combinations of those four components is equally valid. The choice of which to use is a question of convenience in presentation and understanding.

The coefficient $b$ has been adopted since it is a non-dimensional parameter which has a fixed range (between zero and unity) and which can be set to any value between these limits, even in an undrained test. Furthermore the use of $b$ is well rooted in soil mechanics. The effects of $\sigma_{2}$ on the behaviour of soils has been examined in terms of $b$ by many researchers (e.g. Bishop, 1966; Ergun, 1981; Lewin, Yamada \& Ishihara, 1982). The parameter has not been restricted to isotropic soils: it has been used in studying anisotropy at different relative values of the intermediate principal stress, e.g. by Haruyama (1981). In this earlier work, the investigation of $\sigma_{2}$ effects using $b$ was limited to the case of principal stress directions that are fixed during shear. It is logical to extend these investigations to the case of varying principal stress directions while continuing with the use of $b$, provided that $\sigma_{2}$ itself is not rotated.

In the Author's tests in the hollow cylinder apparatus $\sigma_{2}$ does not rotate but is fixed in the horizontal direction. This condition applies to a wide range of field stress paths. Under these conditions the effect of the intermediate principal stress, either in terms of $\sigma_{2}$ or $b$, can be considered independently. If $\sigma_{2}$ had rotated, further parameters would be required to describe fully the state of stress.

Saada and Puccini refer to two series of undrained hollow cylinder tests (described in Saada \& Bianchini (1975)) which they claim illustrate the shortcomings of $b$ as a controlling parameter. In one series of tests $\sigma_{2}$ was kept constant and in the other series the mean stress $p$ was kept constant; in both series the inner and outer pressures $p_{i}$ and $p_{\mathrm{o}}$ were maintained equal throughout. For these conditions, the only difference hetween pairs of tests which are sheared with the same direction for the major principal stress concerns the magnitude of the mean total stress. For saturated samples tested undrained, each pair of tests is therefore exactly equivalent in terms of effective stresses and in the strains that develop. The only difference between each pair of tests is the pore pressure generated, which simply reflects the difference in mean total stress. This is borne out by the results of Saada and Bianchini's tests (see their Fig. 2). Different values of $b$ (and of $\alpha$ since $p_{o}=p_{i}$ ) result in different effective stress paths whereas changing only $\sigma_{2}$ and not $b$ results in the same effective stress path. Surely these re- sults demonstrate an advantage of adopting $b$ rather than $\sigma_{2}$ ?

It is implied in Saada and Puccini's discussion that the decision to use $b$ has necessitated the use of different internal and external pressures. This is not so. Differences between $p_{\mathrm{o}}$ and $p_{\mathrm{i}}$ are necessary to separate the effects of $\sigma_{2}$ from the effects of $\alpha$, independently of the method chosen to express $\sigma_{2}$. In undrained tests on saturated samples this separation can only be achieved with differences between $p_{\mathrm{o}}$ and $p_{\mathrm{i}}$. In drained tests separation of the two effects would be possible with $p_{o}=p_{i}$ but at the expense of varying the mean effective stress $p^{\prime}$.

Referring to the distribution of stresses across the wall of a hollow cylinder specimen, Saada and Puccini have misunderstood the Authors' approach to examining the level of stress nonuniformity. Averages for each stress component have been determined, mainly on the basis of compatibility and equilibrium. These averages have then been compared with averages that would apply if the specimen behaviour was that of different idealized elastic (including anisotropic elastic) and plastic models. The models are not the basis for the stress averages that are used in interpreting data from the hollow cylinder tests, nor are the tests used to establish the validity of these models to describe anisotropic materials.

Saada and Puccini share with many others the common misconception that the use of equal internal and external cell pressures $p_{i}$ and $p_{o}$ produces a uniform distribution of $\sigma_{r}$ and $\sigma_{\theta}$ across the wall of a hollow cylinder specimen. For some forms of anisotropy, stress nonuniformities will arise with $p_{\mathrm{o}}$ and $p_{\mathrm{i}}$ equal, even without a torque applied. Furthermore, even for an isotropic material, stress uniformity cannot be guaranteed when a torque is applied. It is easy to find examples in which such stress nonuniformities arise. Fig. 6(a) shows the distribution of $\sigma_{z}{ }^{\prime}, \sigma_{\theta}{ }^{\prime}, \sigma_{r}{ }^{\prime}$ and $\tau_{\theta z}$ across the wall of a hollow cylinder specimen subjected to torque under undrained conditions with $p_{\mathrm{o}}$ and $p_{\mathrm{i}}$ maintained equal and constant. The specimen was modelled as an isotropic elasto-plastic material; further details of the analysis are given by Gens \& Potts (1984). Fig. 6(b) shows the distribution of stresses for drained application of torque with $p_{o}$ and $p_{i}$ equal. Non-uniformities in all stresses are apparent.

The Authors accept that, with $p_{o}$ different from $p_{i}$, stress non-uniformities exist across the wall of a hollow cylinder specimen. The Authors do not accept that with $p_{\mathrm{o}}=p_{\mathrm{i}}$ uniform conditions necessarily prevail. In developing a philosophy for hollow cylinder testing, the level 
of stress non-uniformities for $p_{o}=p_{i}$ or $p_{o} \neq p_{i}$ must be considered. As described by the $\mathrm{Au}-$ thors, the level of stress non-uniformity is dictated by the geometry of the specimen and by the soil's constitutive relationship. In the $\mathrm{Au}$ thors' opinion, the geometry adopted for their specimen and the bounds which they have set for $p_{\mathrm{o}} / p_{\mathrm{i}}\left(0.9<p_{\mathrm{o}} / p_{\mathrm{i}}<1.2\right)$ limits the stress nonuniformities to acceptable levels. In this respect, it may be of interest to note that in parallel tests conducted by the Authors with $p_{\mathrm{o}}=p_{\mathrm{i}}$ the same overall pattern of behaviour for the sand has been observed (Symes, 1983).

The Authors strongly refute the suggestion that tests run in the hollow cylinder apparatus with $p_{\mathrm{o}} \neq p_{i}$ are 'not much of an improvement over the direct simple shear'. Firstly, the uniformity of stress in a properly dimensioned hollow cylinder specimen subjected to well-chosen combinations of $p_{o}, p_{i}$, torque and axial load is better than in a simple shear device. Secondly, a hollow cylinder apparatus used in this way is a much more versatile tool.

The data presented in the Paper was restricted to those from tests in which $\alpha$ was between $0^{\circ}$ and $45^{\circ}$. This was simply to assist clarity of presentation. The state boundary surface (SBS) has been fully defined in tests with $\alpha$ between $0^{\circ}$ and $90^{\circ}$ (Symes, Shibuya, Hight \& Gens, to be published) and there are no problems with $\alpha>$ $45^{\circ}$.

The concept of the SBS in $q-p^{\prime}-\alpha$ space has also been extended to drained shearing. By normalizing the data in terms of an equivalent pressure to allow for volumetric strains, matching surfaces have been found for drained and undrained behaviour. It is interesting to examine the data presented by Saada and Puccini in Fig. 4 of their discussion in this manner. In view of the dominance of the initial anisotropy that they have demonstrated, the Authors believe that an SBS is applicable. The large differences that are observed in tests at different $\alpha$ do not preclude the possibility of an SBS.

The Authors wish to point out that their tests have not been carried out to establish the full validity of critical state soil models nor to extend these theories. They have carried out a phenomenological study from which has emerged a conceptual framework-the SBS; the SBS is just a component of critical state soil mechanics.

The Authors have shown the value of examining behaviour in relation to the SBS, distinguishing between stress paths on and below the SBS. It should be emphasized that, while pore pressures which develop under undrained conditions will be larger for stress paths travelling on the

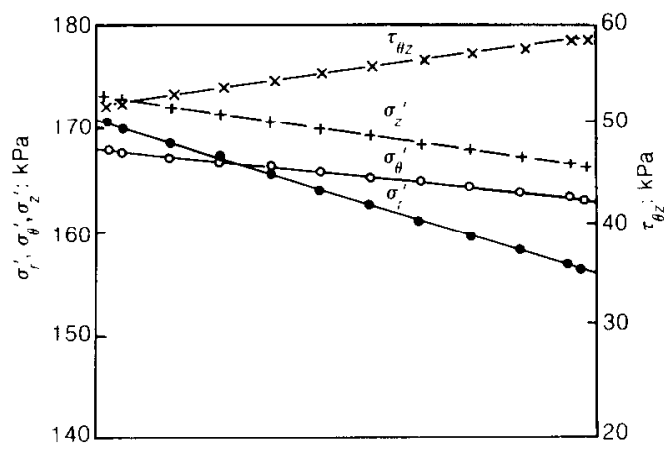

(a)

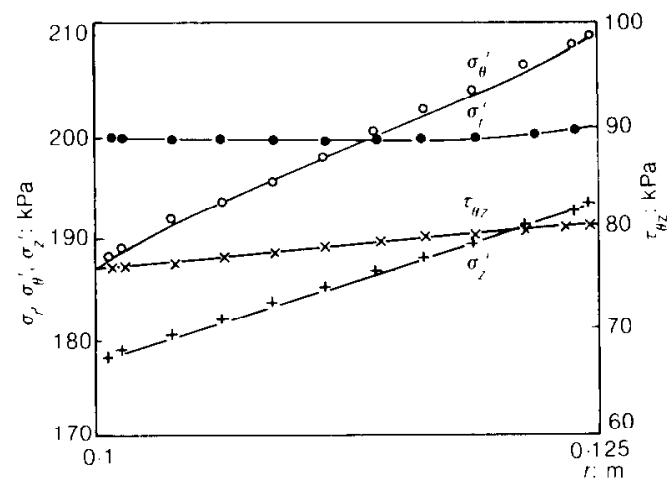

(b)

Fig. 6. Stress distributions across the wall of a hollow cylinder specimen subjected to torque and equal internal and external pressures (isotropic elasto-plastic model): (a) undrained loading; (b) drained loading

SBS, the magnitude of the pore pressures will depend on the orientation of the total stress path relative to the SBS.

The Authors agree with Saada and Puccini that more discussion and research should be directed towards the questions of anisotropy and principal stress rotation so that its practical importance can be determined.

Unfortunately, Dr Jamal begins with the premise that, for equal internal and external pressures in a hollow cylinder test, the radial stress $\sigma_{r}$ is uniformly distributed across the wall of the specimen and is equal to the circumferential stress $\sigma_{\theta}$ irrespective of the shear stress $\tau_{\theta z}$. That this is not necessarily so is demonstrated on Fig. 1 of the Authors' reply to Saada \& Bianchini (1975); even in an isotropic material $\sigma_{r}$ is shown not to be constant and not to be equal to $\sigma_{\theta}$. Only the average values of these stresses, $\bar{\sigma}_{\mathrm{r}}$ and 
$\bar{\sigma}_{\theta}$, are equal; departures from the average values depend on the dimensions of the specimen and on the soil's constitutive relationship.

Jamal's second premise builds on the first and assumes that with $\sigma_{\theta}$ uniformly distributed the circumferential strain $\varepsilon_{\theta}$ is uniform and that $\varepsilon_{\theta}$ is equal to the radial strain $\varepsilon_{r}$. Even when $\sigma_{r}$ and $\sigma_{\theta}$ are equal, $\varepsilon_{\mathrm{r}}$ and $\varepsilon_{\theta}$ can only be guaranteed to be equal in materials which are isotropic in horizontal planes and to which no shear stress $\tau_{\theta z}$ is applied. Although the two strains will remain equal when $\tau_{\theta z}$ is applied to an isotropic elastic material, this will not be the case for most other material constitutive laws.

On this basis, equality of $\varepsilon_{\mathrm{r}}$ and $\varepsilon_{\theta}$ might be anticipated only in the initial part of test E2. In all other stages of the tests which are reported a torque is applied, and the fact that $\varepsilon_{\mathrm{r}}$ is not equal to $\varepsilon_{\theta}$ is not surprising. This conclusion is not affected by the occurrence of volumetric strains.

\section{REFERENCES}

Bishop, A. W. (1966). The strength of soils as en- gineering materials. Géotechnique 16, No. 1, 89130.

Ergun, M. U. (1981). Evaluation of three-dimensional shear testing. Proc. 10th Int. Conf. Soil Mech. Fdn Engng, Stockholm 1, 593-596.

Gens, A. \& Potts, D. M. (1984). Formulation of quasi-axisymmetric boundary value problems for finite element analysis. Engng Comput. 1, June, No. 2, 144-150.

Haruyama, M. (1981). Anisotropic deformationstrength characteristics of an assembly of spherical particles under three-dimensional stresses. Soils Fdns 21, No. 4, 40-55.

Lewin, P. I., Yamada, Y. \& Ishihara, K. (1982). Correlating drained and undrained $3 \mathrm{D}$ tests on loose sand. Proc. IUTAM Conf. Deformation and Failure of Granular Materials, Delft, pp. 419-429.

Saada, A. S. \& Bianchini, G. F. (1975). Strength of one-dimensionally consolidated clays. J. Geotech. Engng Div. Am. Soc. Civ. Engrs 101, GT11, 1151-1164.

Symes, M. J. (1983). Rotation of principal stresses in sand. PhD thesis, University of London.

Symes, M. J., Shibuya, S., Hight, D. W. \& Gens, A. Liquefaction with cyclic principal stress rotation. Proc. 11th Int. Conf. Soil Mech. Fdn Engng, San Francisco, to be published. 